\title{
ON SEMI-PARABOLIC RIEMANN SURFACES
}

\author{
BY
}

\author{
ROBERT D. M. ACCOLA(1)
}

1. Introduction. A bordered Riemann surface whose double is parabolic will be called semi-parabolic. Denote the class of semi-parabolic surfaces by $\mathrm{SO}_{g}$. As usual, a compact bordered surface will be called a finite surface. In a sense, the interiors of semi-parabolic surfaces are the simplest hyperbolic surfaces since their hyperbolicity results entirely from the border which is given in their definition.

On finite surfaces, the class of harmonic functions which are constant on each contour is a finite-dimensional vector space of functions with finite Dirichlet norm. This paper considers the corresponding class of functions on bordered surfaces of class $\mathrm{SO}_{g}$ and generalizes some of the properties of harmonic measures on finite surfaces. In particular, for generalized harmonic measures, we investigate the level curves and their orthogonal trajectories. The principal results, Theorems 4.1 and 4.4, state, in a sense made precise, that almost all of the level curves of a generalized harmonic measure are analytic Jordan curves and almost all of their orthogonal trajectories begin and end on the border given in the definition of the surface. These results have application to the level curves of a Green's function via a theorem of Kuramochi. We also consider the question on a parabolic surface as to when a harmonic differential with finite norm and integral periods is a weak limit of period reproducing differentials.

2. Definitions and notation. If $W$ is a Riemann surface, let $\Gamma(W)$ stand for the Hilbert space of square integrable differentials on $W\left({ }^{2}\right)$. For $\omega, \sigma \in \Gamma(W)$, let $\|\sigma\|_{W}$ denote the norm of $\sigma$, and $(\sigma, \omega)_{W}$ the inner product $\left.{ }^{3}\right)$. Let $\Gamma_{c}$ and $\Gamma_{e}$ denote the closed and exact forms in $\Gamma$. Let $\Gamma_{e o}$ be the closure in $\Gamma_{e}$ of differentials of functions which vanish outside of compact sets. Define $\Gamma_{c o}=\Gamma_{e}^{* \perp}$, $\Gamma_{h}=\Gamma_{c} \cap \Gamma_{c}^{*}, \Gamma_{h o}=\Gamma_{h} \cap \Gamma_{c o}$ and $\Gamma_{h e}=\Gamma_{h} \cap \Gamma_{e}\left({ }^{4}\right) . \Gamma_{h}$ is the Hilbert space of

Presented to the Society, October 22, 1960, under the title Semi-parabolic Riemann surfaces; received by the editors May 3, 1962.

(1) The work on this paper has been conducted over the last three years. During this time the author has been supported by the Office of Ordnance Research, U. S. Army, Contract number DA-19-020-ORD-3779 and by the Office of Naval Research, Contract number Nonr 562 (31).

(2) For a complete discussion of the theory of square integrable differentials see Ahlfors and Sario [1, Chapter V].

(3) In the notation $\Gamma(W),\|\sigma\|_{W}$, and $(\sigma, \omega)_{W}$ the symbol $W$ will be omitted if it is obvious from the context.

(4) $\Gamma_{p}^{*}$ is the set of differentials whose conjugates lie in $\Gamma_{p}$. 
square integrable harmonic differentials on $W$. We then have the following orthogonal decompositions:

$$
\begin{aligned}
& \Gamma=\Gamma_{h}+\Gamma_{e o}+\Gamma_{e o}^{*} \\
& \Gamma_{c}=\Gamma_{h}+\Gamma_{e o} \\
& \Gamma_{c o}=\Gamma_{h o}+\Gamma_{e o} .
\end{aligned}
$$

Let $\Gamma_{h s e}$ stand for the differentials in $\Gamma_{h}$ which have zero periods on all dividing cycles $\left({ }^{5}\right)$. Define $\Gamma_{h m}=\Gamma_{h s e}^{* \perp}\left({ }^{6}\right)$. Define $\Gamma_{h e o}=\Gamma_{h e} \cap \Gamma_{h o}$. Then $\Gamma_{h m} \subset \Gamma_{h e o}$ with equality holding if $W$ is the interior of a finite surface. Note that if $W \subset W^{\prime}$ and $\sigma \in \Gamma_{c o}(W)$, then if we extend $\sigma$ to $W^{\prime}$ to be identically zero in $W^{\prime}-W$ it follows that $\sigma \in \Gamma_{c o}\left(W^{\prime}\right)$. In such a situation we will automatically assume the definition of $\sigma$ so extended.

If $c$ is a cycle we denote by $\sigma(c)$ the unique element of $\Gamma_{h}$ such that for $\omega \in \Gamma_{h}, \int_{c} \omega=\left(\omega, \sigma(c)^{*}\right) . \sigma(c)$ is known to be real, of class $\Gamma_{h o}$, to possess only integral periods and to depend only on the homology class of $c$. We will call $\sigma(c)$ the period reproducer for the cycle $c$, even though the conjugate of $\sigma(c)$ actually reproduces.

If $\sigma$ is a harmonic differential given locally by $a d x+b d y$, we denote by $\rho(\sigma)$ the linear density $\left(|a|^{2}+|b|^{2}\right)^{1 / 2}|d z|\left(^{7}\right)$. If $\rho$ is any linear density then

$$
A_{W}(\rho)=\int_{W} \int \rho^{2} d x d y\left({ }^{8}\right) .
$$

Note that $A_{W}(\rho(\sigma))=\|\sigma\|_{W}^{2}$.

We will use the notation $\bar{W}$ for a bordered Riemann surface; $\bar{W}=W \cup \partial W$ where $W$ is the surface which is the interior of $W$ and $\partial W$ is the border, a countable union of compact and / or noncompact contours. In this context $\hat{W}$ will stand for the double of $\bar{W}$. On a bordered surface $\bar{W}$ we will call a curve $c$ a cross-cut if $c$ is a rectifiable path with end points lying in $\partial W$. If $\sigma$ is a differential in $W$ that can be extended continuously to $\bar{W}$ then $\int_{c} \sigma$ is well defined, and we will call this the cross-cut period of $\sigma$ over $c$. For finite surfaces, differentials of class $\Gamma_{\text {heo }}$ are determined by their cross-cut periods.

If $\bar{W}$ is a bordered surface, $\sigma \in \Gamma_{h}(W)$, and $\sigma$ can be extended to be harmonic on $\bar{W}$, then we will write $\sigma \in \Gamma_{h}(\bar{W})$. If $u$ is a harmonic function on $W$, the subsurface where $u$ takes values between $a$ and $b$ will be denoted by $\{a<u<b\}$.

(5) Ahlfors and Sario [1, p. 66].

(6) In Ahlfors and Sario [1], $\Gamma_{h m}$ is called the set of harmonic measures. In this paper the term "harmonic measure" will be reserved for harmonic functions which take the value zero or one on the boundary of a finite surface. Also all harmonic functions will be considered realvalued, contrary to the usage in Ahlfors and Sario [1, Chapter V].

(7) Ahlfors and Sario [1, p. 220].

(8) We will drop the subscript $W$ if no ambiguity arises. 
If $W$ is a bordered surface we will call a collection $\left\{\bar{\Omega}_{n}\right\}$ of connected finite subsurfaces an exhaustion of $W$ if $\bar{\Omega}_{n}=\bar{\Omega}_{n}^{\prime} \cap W$, where $\left\{\Omega_{n}^{\prime}\right\}$ is an exhaustion of $\hat{W}$ in the usual sense. Thus, the collection $\left\{\overline{\boldsymbol{\Omega}}_{n}\right\}$ doubled across $\partial \Omega_{n} \cap \partial W$ gives a symmetric exhaustion of $\hat{W}$.

3. Preliminary results. We now quote several results which will be necessary for this paper.

Theorem 3.1 (Kuramochi) ${ }^{9}$ ). Suppose $W \subset W^{\prime}$ where $W^{\prime}$ is parabolic and $\partial W$ is a union of piecewise analytic curves in $W^{\prime}$. Then $W \in \mathbf{S O}_{g}$.

THEOREM 3.2. Suppose $W$ is a bordered surface such that $\hat{W} \in O_{H D}$. Then $\sigma \in \Gamma_{h o}(W)$ if and only if $\sigma$ can be extended to be harmonic on $W$ and $\sigma=0$ along $\partial W\left({ }^{10}\right)$.

A simple consequence of this theorem is that if $W \in \mathrm{SO}_{g}\left(\hat{W} \in O_{H D}\right.$ will do) and $d u \in \Gamma_{\text {heo }}(W)$, then $d u$ is uniquely determined by its cross-cut periods.

THEOREM 3.3. Suppose $\rho$ is a linear density on $W$ such that $A(\rho)$ is finite and $W \in \mathrm{SO}_{g}$. Then there exists an exhaustion $\left\{\overline{\boldsymbol{\Omega}}_{n}\right\}$ of $W$, such that $\int_{\partial \Omega_{n} \cap W} \rho \rightarrow 0$ as $n \rightarrow \infty$.

Proof. We extend $\rho$ to be a linear density on $\hat{W}$ by redefining it to be zero on $\partial W$ and defining it to be zero on $\hat{W}-W$. The result follows by the now standard methods of Nevanlinna $\left({ }^{11}\right)$.

\section{4. $\Gamma_{\text {heo }}$ for semi-parabolic surfaces.}

Definition. If $u$ is a harmonic function on a Riemann surface $W$, let $E(u, W)$ be the set of all numbers, $t$, such that some component of the level curves $\{u=t\}$ is noncompact.

If $d u \in \Gamma_{h e}(W)$ where $W$ is a finite surface, then $E(u, W)$ is a subset of the values that $u$ assumes on $\partial W$.

TheOREM 4.1. Suppose $W \in \mathrm{SO}_{g}$ and $d u \in \Gamma_{h o}(W)$. Then the measure of $E(u, W)$ is zero.

Proof. Let $\left\{\bar{\Omega}_{n}\right\}$ be an exhaustion of $W$ such that $\int_{\partial \Omega_{n} \cap W} \rho(d u)=\varepsilon_{n} \rightarrow 0$ as $n \rightarrow \infty$. For technical reasons assume that $\sum \varepsilon_{n}<\infty$. Let $E_{n}$ be the values that $u$ assumes on $\partial \Omega_{n}$. Since $u$ assumes only a finite number of values on $\partial \Omega_{n} \cap \partial W$, the measure of $E_{n}, m\left(E_{n}\right)$, satisfies:

(9) Kuramochi [1]. A fairly simple proof of this can be derived from the method of orthogonal decomposition, Chapter V, Ahlfors and Sario [1].

(10) Accola [2].

(11) Nevanlinna [1]. 


$$
m\left(E_{n}\right) \leqq \int_{\partial \Omega_{n} \cap W}|d u| \leqq \int_{\partial \Omega_{n} \cap W} \rho(d u)=\varepsilon_{n} .
$$

For each $t_{0} \in E(u, W)$, there exists an $n_{0}$ such that $t_{0} \in E_{n}$ for $n \geqq n_{0}$, since for each such $t_{0}$, there is a noncompact component of $\left\{u=t_{0}\right\}$. Therefore, $E(u, W) \subset \bigcup_{k=n}^{\infty} E_{k}$ for all $n$. Thus for all $n: m(E(u, W)) \leqq \sum_{k=n}^{\infty} m\left(E_{k}\right) \leqq \sum_{k=n}^{\infty} \varepsilon_{k}$. q.e.d.

Defining $E(u, \bar{W})$ in an analogous way, it is clear that $E(u, \bar{W})-E(u, W)$ is a countable set if $d u \in \Gamma_{h o}(W)$. Thus $m(E(u, \bar{W}))$ equals zero if $\bar{W}$ is of class $\mathbf{S O}_{g}$.

Kuramochi proved that if $g$ is a Green's function on a surface with pole fixed, then the bordered surface $\{g \geqq \lambda\}$, for $\lambda>0$, is of class $\operatorname{SO}_{g}\left({ }^{12}\right)$. From this and Theorem 1 we obtain the following corollary.

COROLlaRY. If $g$ is a Green's function on a surface with given pole, then the set of $\lambda$, such that the level curves $\{g=\lambda\}$ contain a noncompact component, has measure zero.

Proof. For $\alpha>0$, Kuramochi's result, Theorem 3.1, shows that $\{\alpha \leqq g \leqq \beta\}$ is of class $\mathrm{SO}_{g}$. By Theorem $3.2 \mathrm{dg}$ restricted to $\{\alpha<g<\beta\}$ is of class $\Gamma_{\text {heo }}$. The result now follows easily from Theorem 4.1. q.e.d.

It seems natural to ask whether the property, $m(E(u, W))=0$ characterizes the fact that $d u \in \Gamma_{h e}$ is also in $\Gamma_{h o}$. Theorem 4.1 together with Theorem 3.2 show this to be the case if $W$ can be smoothly embedded in a parabolic surface. In a later paper we will show the characterization to hold if $W$ can be smoothly embedded in a surface where $\Gamma_{h e}$ is finite-dimensional. A surface will also be exhibited to show that the property does not, in general, characterize. One half of the desired characterization is true, however.

THEOREM 4.2. Let $W$ be an arbitrary Riemann surface. Suppose $d u \in \Gamma_{h e}(W)$ and the measure of $E(u, W)$ is zero. Then $d u \in \Gamma_{h o}(W)$.

Proof. Define an equivalence relation on the connected components of the level curves of $u$ as follows. If a component is noncompact or has a point where the gradient of $u$ vanishes, it will be equivalent to itself only. Call such components irregular. All other components, which we call regular, are analytic Jordan curves. Two such components will be equivalent if they bound an annulus in $W$. It is readily seen that this is an equivalence relation. Moreover, if a component is regular, it follows by an easy compactness argument that there are many other components equivalent to it. Let $A$ be a generic notation for the union of all regular level curves in an equivalence class. Let $A_{n}, n=1,2, \cdots$, be an enumeration of the $A$ 's. Each $A_{n}$ is seen to be an annulus.

If $\left\{\Omega_{n}\right\}$ is an exhaustion of $W$ we see that

(12) Kuramochi [2]. 


$$
\|d u\|_{\Omega_{n}}^{2}=\int_{-\infty}^{\infty} d k \int_{\{u=k\} \cap \Omega_{n}} d u^{*}
$$

where the level curves $\{u=k\}$ are oriented so that $d u^{*}$ is positive. Letting $n \rightarrow \infty$, it follows that $\|d u\|_{W}^{2}=\int_{-\infty}^{\infty} d k \int_{\{u=k\}} d u^{*}$. If we let $F=(-\infty, \infty)-$ $(E(u, W) \cup S)$ where $S$ is the set of all $t$ such that $\{u=t\}$ contains a branched level curve, then $\|d u\|_{W}^{2}=\int_{F} d k \int_{\{u=k\}} d u^{*}$ since the complementary set is of measure zero. But every point in the set over which this last double integral is evaluated is on a regular level curve. Thus $\|d u\|_{W}^{2} \leqq \sum_{n=1}^{\infty}\|d u\|_{A_{n}}^{2}$ and so $\|d u\|^{2}=\sum_{n=1}^{\infty}\|d u\|_{A_{n}}^{2}$.

If $d u_{n}$ denotes the restriction of $d u$ to $A_{n}$ then $d u_{n} \in \Gamma_{h m}\left(A_{n}\right)$ and so $d u_{n}$, suitably extended, is of class $\Gamma_{c o}(W)$. Since $d u=\sum d u_{n}$ the result now follows. q.e.d.

Since each $A_{n}$ is an annulus, it follows that we can find a sequence $\left\{\Omega_{n}\right\}$ where

(1) each $\Omega_{n}$ is a finite union of relatively compact annuli;

(2) $\bar{\Omega}_{n} \subset \Omega_{n+1}$;

(3) if $A_{n}^{j}, j=1,2, \cdots, r_{n}$ is an enumeration of the annuli in $\Omega_{n}$, and $u_{n}^{j}$ denotes $u$ restricted to $A_{n}^{j}$, then $d u_{n}^{j} \in \Gamma_{h m}\left(A_{n}^{j}\right)$;

$$
\left\|d u_{n}-d u\right\| \rightarrow 0 \quad \text { where } \quad d u_{n}=\sum_{j=1}^{r_{n}} d u_{n}^{j} .
$$

Thus, in a certain sense, any $d u \in \Gamma_{h e}$ with $E(u, W)=0$ can be approximated by harmonic measures on unions of finite subsurfaces. This is unsatisfactory, however, since the $\Omega_{n}$ 's above are neither connected nor do they exhaust $W$. If $W$ is the interior of a semi-parabolic surface, we can make a stronger approximation statement. We need several lemmas preliminary to Theorem 4.3.

LEMMA 1. Let $u$ be a harmonic function such that $d u \in \Gamma_{h e}(W)$. Let $s$ be the function such that $s=a$ on $\{u \leqq a\}, s=u$ on $\{a \leqq u \leqq b\}$ and $s=b$ on $\{u \geqq b\}$, where $a<b$. Then $d s \in \Gamma_{e}(W)$.

Proof. Omitted.

Lemma 2. Suppose $d u \in \Gamma_{h e}(\bar{W})$ where $\bar{W}$ is a finite surface. Define $\sigma$ so that $\sigma=d u$ for points $p$ such that $u(p) \notin E(u, W)$ and $\sigma=0$ for points $p$ such that $u(p) \in E(u, W)$. Then $\sigma \in \Gamma_{c o} \cap \Gamma_{e}(W)$.

Proof. $\sigma$ is a finite union of differentials of the type $d s$ considered in Lemma 1 since the range of $u$ minus $E(u, W)$ is the union of a finite number of open intervals. $\sigma \in \Gamma_{c o}(W)$ since it vanishes along $\partial W$.

Lemma 3. Let $\bar{W}$ be a bordered surface. Suppose $\partial W=B_{0} \cup B_{1}$ where $B_{0} \cap B_{1}=\varnothing$ and each $B_{i}$ is homeomorphic to an open interval. Suppose $p_{i} \in B_{i}$ and let $c$ be a cross-cut joining $p_{0}$ to $p_{1}$. Suppose $\omega \in \Gamma_{c}(\bar{W})$ so that $\omega=0$ along $\partial W$, and assume $\omega$ is harmonic in a neighborhood of $\partial W$. Let $\sigma$ be the projection of $\omega$ on $\Gamma_{h}(W)$. Then $\sigma \in \Gamma_{h}(\bar{W}), \sigma=0$ along $\partial W$ and $\int_{c} \sigma=\int_{c} \omega$. 
Proof. Let $\tilde{\omega}$ be the anti-symmetric extension of $\omega$ to $\hat{W}\left({ }^{13}\right)$. Let $\tilde{\omega}=\tilde{\sigma}+\tilde{\tau}$, $\tilde{\sigma} \in \Gamma_{h}(\hat{W}), \tilde{\tau} \in \Gamma_{e o}(\hat{W})$, be the orthogonal decomposition of $\tilde{\omega} \in \Gamma_{c}(\hat{W})$. By the uniqueness of the decomposition it follows that $\tilde{\sigma}$ and $\tilde{\tau}$ are anti-symmetric. If $\sigma$ and $\tau$ are the restrictions of $\tilde{\sigma}$ and $\tilde{\tau}$ to $W$ then it follows that $\tau \in \Gamma_{e o}(W)\left({ }^{14}\right)$. Thus $\omega=\sigma+\tau$ is the orthogonal decomposition of $\omega$ in $\Gamma_{c}(W)$. The first two parts of the conclusion now follow. If $\tilde{c}$ is the path $c-j c$, where $j$ is the natural reflection in $\hat{W}$, then $\int_{\tilde{c}} \tilde{\omega}=\int_{\tilde{c}} \tilde{\sigma}$. But $\int_{\tilde{c}} \tilde{\omega}=2 \int_{c} \omega$ and $\int_{\tilde{c}} \tilde{\sigma}=2 \int_{c} \sigma$. q.e.d.

TheOREM 4.3. Suppose $W \in \mathrm{SO}_{g}$ and $d u \in \Gamma_{\text {heo }}(W)$. Then there exists an exhaustion $\left\{\bar{\Omega}_{n}\right\}$ of $\bar{W}$ and $d u_{n} \in \Gamma_{\text {heo }}\left(\Omega_{n}\right)$ such that $\left\|d u_{n}-d u\right\| \rightarrow 0$ as $n \rightarrow \infty$.

Proof. Choose $\left\{\Omega_{n}\right\}$, an exhaustion of $W$, so that $\varepsilon_{n}=\int_{\partial \Omega_{n} \cap W} \rho(d u) \rightarrow 0$ as $n \rightarrow \infty$. On $\Omega_{n}$ define the differential $\sigma_{n}$ as follows. For each $p \in \Omega_{n}$ let $\sigma_{n}=d u$ if $u(p) \notin E\left(u, \Omega_{n}\right)$ and let $\sigma_{n}=0$ if $u(p) \in E\left(u, \Omega_{n}\right)$. Then by Lemma $2, \sigma_{n} \in \Gamma_{c o}$ $\cap \Gamma_{e}\left(\Omega_{n}\right) \subset \Gamma_{c o}(W)$. Moreover, $\left\|\sigma_{n}\right\|<\|d u\|$. Now let $d u_{n}$ be the projection of $\sigma_{n}$ on $\Gamma_{h}\left(\Omega_{n}\right)$. It follows that $d u_{n} \in \Gamma_{h e o}\left(\Omega_{n}\right) \subset \Gamma_{c o}(W)$. By Lemma 3, $\sigma_{n}$ and $d u_{n}$ have the same cross-cut periods and $\left\|d u_{n}\right\|<\left\|\sigma_{n}\right\|<\|d u\|$. As $n \rightarrow \infty$ the cross-cut periods of $\sigma_{n}$ over a fixed cross-cut approach that of $d u$ because $\varepsilon_{n} \rightarrow 0$. Since the $d u_{n}$ 's are uniformly bounded in norm, a subsequence converges in $\Gamma_{c o}(W)$ to an exact harmonic differential with the same cross-cut periods as $d u$. Thus $d u_{n} \rightarrow d u$ weakly in $\Gamma_{c o}$. Moreover, lim sup $\left\|d u_{n}\right\| \leqq\|d u\| \leqq \lim$ inf $\left\|d u_{n}\right\|$; that is, $\left\|d u_{n}\right\| \rightarrow\|d u\|$ and so the convergence is strong. q.e.d.

We now raise the question as to the nature of the trajectories orthogonal to the level curves of a function $u$ where $d u \in \Gamma_{h o} \cap \Gamma_{h e}$. We first give a definition.

Definition. Let $u$ be harmonic on $W$ (or $\bar{W}$ ). Define two points $p$ and $q$ to be equivalent if they can be joined by a piecewise analytic curve over any subarc of which $d u^{*}$ has zero integral. The equivalence classes will be defined as the orthogonal trajectories of $u$.

Let $\gamma$ be a compact connected subset of $\partial W$ where $W \in \mathrm{SO}_{g}$. Suppose $u$ is harmonic on $W \cup \gamma, u$ positive on $W$ and $u=0$ on $\gamma$. Parametrize $\gamma$ by a function $f$ defined on an interval $[0, a]$ so that $s=\int_{f(0)}^{f(s)} d u^{*}$ and $a=\int_{\gamma} d u^{*}$ where $\gamma$ is oriented so that $a$ is positive. Let $l(s)$ be the orthogonal trajectory of $u$ passing through $f(s)$. Since there are a countable number of points in $W$ where the gradient of $u$ is zero, $l(s) \cap W$ will be homeomorphic to a line except for at most a countable number of values of $s$. Call a value of $s$ regular if $l(s)$ is unbranched and $l(s)$ is relatively compact in $\hat{W}$. Call other values of $s$ irregular.

THEOREM 4.4. Under the hypotheses of the preceding paragraph, the set of irregular s has measure zero if either (a) $u$ is bounded or (b) $u$ is Dirichlet bounded.

(13) Ahlfors and Sario [1, p. 290].

(14) Ibid., p. 288. This follows from Lemma $13 b$. 
Proof. Let $F$ be the set of irregular $s . F$ is measurable since the set of regular $s$ is open. Let $F^{\prime}$ be the set of $s$ in $F$ such that $l(s)$ is unbranched. Assuming the measure, $b$, of $F^{\prime}$ is positive, we will show that the extremal distance from $\gamma$ to the ideal boundary, $\infty$, in $\hat{W}$ is finite, contradicting the characterization of Ohtsuka $\left({ }^{1}\right.$ ) which states that this distance is infinite if $\hat{W} \in O_{g}$.

(a) Assume first that $u \leqq M$ on $W$. Let $\rho$ be any linear density on $W$. Then $L(\rho)^{2} \leqq\left|\int_{l(s)} \rho\right|^{2}$ for any $s \in F^{\prime}$ where $L(\rho)$ is the minimum $\rho$-length of a curve going from $\gamma$ to $\infty$ in $\hat{W} . l(s)$ still denotes the orthogonal trajectory of $u$ in $W \subset \hat{W}$. By the Schwarz inequality

$$
L(\rho)^{2} \leqq \int_{l(s)} \rho^{2} \int_{l(s)} d u \leqq M \int_{l(s)} \rho^{2} .
$$

Integrating this inequality over $F^{\prime}$ with respect to $d u^{*}$ yields

$$
b L(\rho)^{2} \leqq \int_{F^{\prime}}\left(M \int_{l(s)} \rho^{2}\right) d u^{*} \leqq M A(\rho) .
$$

Thus $L(\rho)^{2} / A(\rho) \leqq M / b$ for all $\rho$. Thus $b>0$ leads to the desired contradiction since $F-F^{\prime}$ is countable.

(b) In case $u$ is unbounded, the result follows by exhausting $\bar{W}$ by the subsurfaces $\{0 \leqq u \leqq n\}$ as $n \rightarrow \infty$. Since the norm of $d u$ is assumed to be bounded, the set of $s$ such that $u$ is unbounded on $l(s)$ must have measure zero. The result now follows. q.e.d.

The example of $y$ on $\{y \geqq 0\}$ shows that, in general, either assumption (a) or (b) must hold for the conclusion to follow.

Definition (Heins). If $u$ is harmonic and the greatest harmonic minorant of $u$ and $1-u$ is zero, then $u$ will be called a generalized harmonic measure.

Suppose $\bar{W} \in \mathrm{SO}_{g}, \partial W=B_{0} \cup B_{1}$, where $B_{0} \cap B_{1}=\varnothing$, and each $B_{i}$ is a union of contours. Suppose, moreover, that $u$ is harmonic on $W, u=0$ on $B_{0}, u=1$ on $B_{1}$ and $0 \leqq u \leqq 1$ on $\bar{W}$. Then $u$ is a generalized harmonic measure on $W$. Let $\left\{\alpha_{i}\right\}$ be an enumeration of the components of $B_{0}$. If $\alpha_{i}$ is compact, parametrize it by a function $f_{i}$ defined on an interval, $I_{i},\left[0, a_{i}\right]$ so that for $s \in I_{i}$, $s=\int_{f_{i}(0)}^{f_{i}(s)} d u^{*}$ and $a_{i}=\int_{\alpha_{i}} d u^{*}$. If $\alpha_{i}$ is noncompact, parametrize it by a function $f_{i}$ defined on an open interval $I_{i},\left(0, a_{i}\right),(0, \infty),(-\infty, 0)$ or $(-\infty, \infty)$ so that $s_{2}-s_{1}=\int_{f\left(s_{1}\right)}^{f\left(s_{2}\right)} d u^{*}$ for $s_{1}, s_{2} \in I_{i}$. If there is an $I_{i}$ of the type $(-\infty, \infty)$, $(0, \infty)$, or $(-\infty, 0)$ then $\int_{B_{0}} d u^{*}=\infty$. Otherwise, $\int_{B_{0}} d u^{*}=\sum a_{i} \leqq \infty$.

COROLLARY 4.4.1. Under the hypotheses of the preceding paragraph, the set of irregular $s$ in all parametric intervals has measure zero.

Proof. Each parametric interval can be divided into a countable number of compact intervals of the type considered in Theorem 4.4 .

(15) Ohtsuka [1]. 
CoROLlary 4.4.2. Assuming the hypotheses of the paragraph preceding Corollary 4.4.1, let $k$ be any number between zero and one. Then $\int_{\{u=k\}} d u^{*}=\int_{B_{0}} d u^{*}$ where $\{u=k\}$ is parametrized in the same manner as above.

Proof. It suffices to prove this for $k=1$. Let $J_{i}^{j}$ be an enumeration of the disjoint open intervals of $I_{i}$ whose union is the set of regular values in $I_{i}$. We assert that the end points in $B_{1}$ of all $l(s)$ such that $s$ lies in $J_{i}^{j}$, for fixed $i$ and $j$, lie in the same component of $B_{1}$. For if $s_{0}$ is in $J_{i}^{j}$, let $J^{\prime}$ be the set of all $s \in J_{i}^{j}$ such that the end points of $l(s)$ and $l\left(s_{0}\right)$ lie in the same component of $B_{1} . J^{\prime}$ and $J_{i}^{j}-J^{\prime}$ are seen to be open and so the assertion is proven. In fact, $\bigcup_{s \in J_{i}^{j}}^{j}(s)$ is conformally equivalent to a rectangle of dimension $a_{i}^{j}$ by one where $a_{i}^{j}$ is the length of $J_{i}^{j}$. The parameter $z=\int_{p_{0}}^{p} d u+i d u^{*}$ effects the conformal map.

Since points of $B_{1}$ which are end points of regular orthogonal trajectories, $l(s)$, for $s \in J_{i}^{j}$ are end points of no other $l(s)$ we see that $\int_{\{u=1\}} d u^{*} \geqq \int_{B_{0}} d u^{*}$. Arguing with $1-u$ instead of $u$ gives the desired equality. q.e.d.

Concerning generalized harmonic measures on bordered surfaces of class $\mathrm{SO}_{g}$, Theorems 4.1 and 4.4 together with the latter's corollaries seem to be satisfactory generalizations of the standard facts about level curves and their orthogonal trajectories for harmonic measures on finite surfaces.

5. Period reproducers. Differentials of harmonic measures on finite surfaces are period reproducers for dividing cycles. We now investigate how this fact generalizes to bordered surfaces of class $\mathrm{SO}_{g}$.

TheOREM 5.1. Suppose $\bar{W} \in \mathrm{SO}_{g} . \partial W=B_{0} \cup B_{1}$, where $B_{0} \cap B_{1}=\varnothing$ and each $B_{i}$ is a union of contours. Suppose $d u \in \Gamma_{h e}(\bar{W}), u=0$ on $B_{0}$ and $u=1$ on $B_{1}$. For any $k$ between zero and one, orient the components of $\{u=k\}$ so that $d u^{*}$ is positive. Then

(1) $\int_{\{u=k\}} d u^{*}=\|d u\|^{2}$ for all $k$;

(2) if $\sigma \in \Gamma_{h}(W)$ then $\int_{\{u=k\}} \rho(\sigma)$ converges for almost all $k$, and for these $k$;

(3) $\int_{\{u=k\}} \sigma=\left(\sigma, d u^{*}\right)$.

Proof. We first prove that $\int_{\{u=k\}} d u^{*}$ is a finite constant independent of $k$ by methods which seem simpler than those used in Corollary 4.4.2. 'As in the proof of Theorem 4.2 we see that

$$
\|d u\|^{2}=\int_{0}^{1} d k \int_{\{u=k\}} d u^{*}
$$

Thus for almost all $k$ we have: $\int_{\{u=k\}} d u^{*}<\infty$. Let $\left\{\bar{\Omega}_{n}\right\}$ be an exhaustion of $W$ such that $\int_{\partial \Omega_{n} \cap W} \rho(d u) \rightarrow 0$ as $n \rightarrow \infty$. Suppose $\int_{\{u=\alpha\}} d u^{*}$ is finite and $\beta \neq \alpha$. Since $d u^{*}$ is closed we have $\int \partial\left(\{\alpha \leqq u \leqq \beta\} \cap \Omega_{n}\right) d u^{*}=0$. Thus 


$$
0=-\int_{\{u=\alpha\} \cap \Omega_{n}} d u^{*}+\int_{\{u=\beta\} \cap \Omega_{n}} d u^{*}+\int_{\partial \Omega_{n} \cap\{\alpha \leqq u \leqq \beta\}} d u^{*} .
$$

As $n \rightarrow \infty$, the third term approaches zero and so $\int_{\{u=\beta\}} d u^{*}$ converges to $\int_{\{u=\alpha\}} d u^{*}$. Thus $\int_{\{u=k\}} d u^{*}$ converges for all $k$ to the same limit. That the limit is $\|d u\|^{2}$ follows immediately from $(*)$.

For part (2) we proceed as in the proof of Theorem 4.2 to obtain

$$
\|\sigma\|^{2}=A(\rho(\sigma))=\int_{0}^{1} d k \int_{\{u=k\}} \rho(\sigma)^{2} d u^{*} .
$$

Thus for almost all $k, \int_{\{u=k\}} \rho(\sigma)^{2} d u^{*}$ converges. But

$$
\left(\int_{\{u=k\}} \rho(\sigma) d u^{*}\right)^{2} \leqq\left(\int_{\{u=k\}} d u^{*}\right)\left(\int_{\{u=k\}} \rho(\sigma)^{2} d u^{*}\right) .
$$

Thus $\int_{\{u=k\}} \rho(\sigma) d u^{*}$ converges for almost all $k$, and, therefore, for the same $k$ $\int_{\{u=\alpha\}} \sigma$ converges.

For part (3) we see in exactly the same way as in the proof of part (1) that if $\int_{\{u=\alpha\}} \sigma$ and $\int_{\{u=\beta\}} \sigma$ converge then the limits are the same. Call this limit $L$. By Green's formula we have

$$
\left(\sigma, d u^{*}\right)_{\{\alpha<u<\beta\} \cap \Omega_{n}}=-\alpha \int_{\{u=\alpha\} \cap \Omega_{n}} \sigma+\beta \int_{\{u=\beta\} \cap \Omega_{n}} \sigma+\int_{\partial \Omega_{n} \cap\{\alpha \leqq u \leqq \beta\}} u \sigma .
$$

Choosing an exhaustion $\left\{\bar{\Omega}_{n}\right\}$ so that $\int_{\partial \Omega_{n} \cap W} \rho(\sigma) \rightarrow 0$ as $n \rightarrow \infty$, and observing that $0<u<1$, we see that the third term of the right hand side of the last equation approaches zero as $n \rightarrow \infty$. Assuming that the integrals of $\sigma$ over $\{u=\alpha\}$ and $\{u=\beta\}$ converge we see that $\left(\sigma, d u^{*}\right)_{\{\alpha<u<\beta\}}=(\beta-\alpha) L$. Letting $\alpha \rightarrow 0$ and $\beta \rightarrow 1$ proves part (3). q.e.d.

If $W$ is a finite surface then $\{u=k\}$ is compact. In case there is a value of $k$ so that $\{u=k\}$ is compact in the general situation of Theorem 5.1 , then it is easy to show that in fact $d u$ is the reproducer for this cycle. In general, however, $\{u=k\}$ will be noncompact for every $k$. We have been unable to prove in this general situation that one value of $k$ will serve for all $\sigma \in \Gamma_{h}$ in part (3) of the statement of the last theorem. If this were the case, and if $\{u=k\}$ were a union of compact components, $A_{1}, A_{2}, \cdots$, then there would exist a sequence of cycles, $c_{n}=\sum_{k=1}^{n} A_{k}$ such that $\sigma\left(c_{n}\right) \rightarrow d u$ weakly in $\Gamma_{h}(W)$. One might hope for the weaker result, that there exists some sequence of cycles $c_{n}$ so that $\sigma\left(c_{n}\right) \rightarrow d u$ weakly. Although we have no counter-example, we believe this is not in general true. In the presence of some metric and/or topological restrictions on the surface, the theorem is true. The following strong restriction does cover the example exhibited in Accola $[2$, p. 159]. We have no doubt that there are other such restrictions which insure the result of Theorem 5.2.

Definition. Let $W$ be a Riemann surface, $\Omega_{0}$ a fixed finite subsurface. Let $\Delta_{N}$ 
be the set of all $\partial \Omega$ such that: (1) $\Omega_{0} \subset \Omega$; (2) $\bar{\Omega}$ is a finite surface in $W$; and (3) $\partial \Omega$ has $N$ or less components. If the extremal length of the family $\Delta_{N}$ is zero for some $N<\infty$, we will say that $W$ satisfies condition $X$.

Note that the definition implies that $W$ is a parabolic surface and has $N$ or less ideal boundary points. The property is independent of $\Omega_{0}$ choosen for purposes of the definition.

THEOREM 5.2. Let $\bar{W}\left(\in \mathrm{SO}_{g}\right)$ be a bordered surface which can be embedded in a parabolic surface, $W^{\prime}$ which satisfies condition $X$ so that $\partial W$ is piecewise analytic. Let $\partial W=B_{0} \cup B_{1}$, where $B_{0} \cap B_{1}=\varnothing$ and $B_{0}$ and $B_{1}$ are unions of contours. Let $u$ be harmonic on $W, u=i$ on $B_{i}, 0 \leqq u \leqq 1$, and $\|d u\|<\infty$. Then there exists a sequence of cycles $c_{n}$ such that $\sigma\left(c_{n}\right) \rightarrow d u$ weakly.

Proof. Extend $\rho(d u)$ to be zero on $W^{\prime}-W$. Then there exists an exhaustion $\left\{\Omega_{n}^{\prime}\right\}$ of $W^{\prime}$ such that $\partial \Omega_{n}^{\prime}$ has $N$ or less components and as $n \rightarrow \infty$ we have:

$$
\varepsilon_{n}=\int_{\partial \Omega_{n}^{\prime}} \rho(d u) \geqq \int_{\partial \Omega_{n}^{\prime} \cap W} \rho(d u) \rightarrow 0 .
$$

Assume $\varepsilon_{n} \leqq 1 / 2$. Let $\Omega_{n}=\Omega_{n}^{\prime} \cap W$ and let $\bar{\Omega}_{n}$ be the finite set of finite surfaces of which the components of $\Omega_{n}$ are the interiors.

The range of $u$ restricted to $\partial \Omega_{n}$ is a finite set of closed intervals, some possibly degenerate, lying in the unit interval. We show that there are at most $N+2$ such closed intervals. If the image under $u$ of a contour, $\alpha$, of $\partial \Omega_{n}$ lies in $(0,1)$ then $\alpha$ lies in $W$ and is a contour of $\partial \Omega_{n}^{\prime}$. Therefore, there are at most $N$ such $\alpha$ 's. The other contours of $\partial \Omega_{n}$ intersect $B_{0}$ or $B_{1}$ and so their images contain zero or one. Thus there are at most $N+2$ such intervals. The total length of the image of $\partial \Omega_{n}$ under $u$, which is less than the variation of $u$ on $\partial \Omega_{n}$, is less than or equal to $\varepsilon_{n}$ since on $B_{0}$ or $B_{1}, u$ takes the value zero or one. Thus the image of $\partial \Omega_{n}$ under $u$ is at most $N+2$ closed intervals of total length at most $\varepsilon_{n}$.

The complementary set in $[0,1]$ is at most $N+1$ open intervals of total length greater than or equal to $1-\varepsilon_{n}$. Thus there exists a complementary open interval of length greater than or equal to $\left(1-\varepsilon_{n}\right) /(N+1)$. We can, therefore, divide $\partial \Omega_{n}$ into two sets $\alpha_{n}$ and $\beta_{n}$ so that $\alpha_{n}$ and $\beta_{n}$ are unions of contours of $\partial \Omega_{n}$ and for $p \in \alpha_{n}$ and $q \in \beta_{n}, \quad u(p)-u(q) \geqq\left(1-\varepsilon_{n}\right) /(1+N) \geqq 1 /(2(1+N))$ since $\varepsilon_{n}<1 / 2$. In $\bar{\Omega}_{n}$ define a harmonic function $u_{n}$ so that $u_{n}=1$ on $\alpha_{n}$ and $u_{n}=0$ on $\beta_{n}$. Since $\bar{\Omega}_{n}$ is a union of finite surfaces, $d u_{n}$ is a period reproducer in $\Omega_{n}$ for some cycle $c_{n}$; in fact, $c_{n}$ is homologous to $\alpha_{n}$ if the contours of $\alpha_{n}$ are suitably oriented. We now obtain a bound on $\left\|d u_{n}\right\| \cdot\left\|d u_{n}\right\|^{-2}$ is the extremal distance in $\bar{\Omega}_{n}$ between $\alpha_{n}$ and $\beta_{n}\left({ }^{16}\right)$. From the manner in which $\alpha_{n}$ and $\beta_{n}$ were chosen it follows that the minimum $\rho(d u)$ distance between $\alpha_{n}$ and $\beta_{n}$ is $\geqq 1 /(2(1+N))$. Thus:

$$
\left\|d u_{n}\right\|^{-2} \geqq\left(2(N+1)\|d u\|_{\Omega_{n}}\right)^{-2} \geqq(2(N+1)\|d u\|)^{-2}
$$

(16) Ahlfors and Sario [1]. This is still true for $\Omega_{n}$ disconnected. 
Thus $\left\|d u_{n}\right\| \leqq 2(N+1)\|d u\|$.

Since $\left\{\left\|d u_{n}\right\|\right\}$ is a bounded sequence of numbers, $d u_{n}$ converges weakly in $\Gamma_{c o}(W)$ to an exact harmonic differential with the same cross-cut periods as $d u$; i.e., $d u_{n}$ converges weakly to $d u$. But for $\sigma \in \Gamma_{h}(W),\left(\sigma, d u_{n}^{*}\right)=\int_{c n} \sigma$ $=\left(\sigma, \sigma\left(c_{n}\right)^{*}\right)$ where $\sigma\left(c_{n}\right) \in \Gamma_{h o}(W)$. Thus $\sigma\left(c_{n}\right) \rightarrow d u$ weakly. q.e.d.

We now discuss differentials with integral periods on parabolic surfaces. If $\sigma=\sigma(c)$ for some cycle then $\sigma$ has integral periods. Also if $\sigma$ has integral periods and $W$ is a closed surface then $\sigma$ is a reproducer for some cycle.

Suppose $W$ is parabolic and $\sigma \in \Gamma_{h}(W)$ has integral periods. By the methods outlined in Accola [1] we can divide $W$ into a countable number of bordered subsurfaces $W^{i}$, such that: (1) the $W^{i}$ s are mutually disjoint; (2) $\sigma$ restrivted to $W^{i}$ is exact; call it $d u^{i}$, and $u^{i}$ can be chosen so that $0 \leqq u^{i} \leqq 1$; (3) $\partial W^{i}=\alpha^{i} \cup \beta^{i}$, where $\alpha^{i}$ and $\beta^{i}$ are unions of contours and $u^{i}=1$ on $\alpha^{i}$ and $u^{i}=0$ on $\beta^{i}\left({ }^{17}\right)$. By the result of Kuramochi each $W^{i}$ is semi-parabolic. Thus $d u^{i}$ on $W^{i}$ is a differential of the type considered in Theorem 5.1 and is, therefore, in the sense of that theorem a period reproducer for a family of infinite cycles. If $W$ is a surface which satisfies condition $X$ then a stronger statement is possible.

THEOREM 5.3. Let $W\left(\in O_{g}\right)$ be a surface which satisfies condition $X$. If $\sigma \in \Gamma_{h}(W)$ has integral periods then there exists a sequence of cycles $c_{n}$, such that $\sigma\left(c_{n}\right) \rightarrow \sigma$ weakly.

Proof. Divide $W$ into subsurfaces $W^{i}$ and let $\sigma=d u^{i}$ in each $W^{i}$ as described above. Let $\left\{\Omega_{n}\right\}$ be an exhaustion of $W$ such that $\varepsilon_{n}=\int_{\partial \Omega_{n}} \rho\left(^{\sigma}\right) \rightarrow 0$ as $n \rightarrow \infty$, $\varepsilon_{n}<1 / 2$, and $\partial \Omega_{n}$ has $N$ or less components. Fixing the index $i$ and letting $W^{i}, W$ and $\Omega_{n}$ play the roles of $W, W^{\prime}$, and $\Omega_{n}^{\prime}$ in Theorem 5.2, we obtain a sequence of differentials $d u_{n}^{i} \in \Gamma_{c o}\left(W^{i}\right)$ and cycles $c_{n}^{i}$ such that: (1) $d u_{n}^{i} \rightarrow d u^{i}$ weakly in $\Gamma_{c o}\left(W^{i}\right)$; (2) the cross-cut periods of $d u_{n}^{i}$ are eventually those of $d u^{i}$; (3) for $\tau \in \Gamma_{h}\left(W^{i}\right),\left(\tau, d u_{n}^{i *}\right)=\int_{c_{n}^{i}} \tau ;$ and

$$
\left\|d u_{n}^{i}\right\|_{W^{i}}^{2} \leqq 4(N+1)^{2}\left\|d u^{i}\right\|_{W^{i}}^{2}=4(N+1)^{2}\|\sigma\|_{W^{i}}^{2}
$$

If $\Omega_{n} \cap W^{i}=\varnothing$ set $d u_{n}^{i}=0$ and choose $c_{n}^{i}$ to be a curve homologous to zero. Let $c_{n}=\sum_{i=1}^{\infty} c_{n}^{i}$ and let $\omega_{n}=\sum_{i=1}^{\infty} d u_{n}^{i}\left({ }^{18}\right)$. Then

$$
\left\|\omega_{n}\right\|^{2} \leqq 4(N+1)^{2} \sum_{i=1}^{\infty}\|\sigma\|_{W^{i}}^{2}=4(N+1)^{2}\|\sigma\|^{2} .
$$

Also, for $\tau \in \Gamma_{h}(W),\left(\tau, \omega_{n}^{*}\right)=\int_{c_{n}} \tau$. For a fixed cycle $\gamma$ and $n$ so large that the support of $\gamma$ is included in $\Omega_{n}$, it follows that $\int_{\gamma} \omega_{n}=\int_{\gamma} \sigma$ since $\int_{\gamma} \sigma$ is determined by the manner in which $\gamma$ intersects the $\bar{W}^{i}$. Since $\omega_{n} \in \Gamma_{c o}(W)$ reproduces for

(17) The proof in Accola [1], was for closed surfaces but it works equally well for parabolic or semi-parabolic surfaces with the obvious modifications.

(18) There may be only a finite number of $W_{i}$, in which case this is to be taken as a finite sum. 
$c_{n}, \sigma\left(c_{n}\right)$ is the projection of $\omega_{n}$ on $\Gamma_{h}(W)$, and so $\sigma\left(c_{n}\right)$ has the same periods as $\omega_{n}$. It follows that $\sigma\left(c_{n}\right) \rightarrow \sigma$ weakly since the $\sigma\left(c_{n}\right)$ are uniformly bounded in norm. q.e.d.

R. D. M. Accola

\section{REFERENCES}

1. Differentials and extremal length on Riemann surfaces, Proc. Nat. Acad. Sci. U.S.A. 46 (1960), 540-543.

2. The bilinear relation on open Riemann surfaces, Trans. Amer. Math. Soc. 96 (1960), 143-161.

L. Ahlfors ANd L. SARio

1. Riemann surfaces, Princeton Univ. Press, Princeton, N. J., 1960.

Z. KURAMOCHI

1. On covering surfaces, Osaka Math. J. 5 (1953), 155-201.

2. Capacity of subsets of the ideal boundary, Proc. Japan Acad. 32 (1956), 111-116.

R. NeVANLINNA

1. Quadratisch Integrierbare Differentiale auf einer Riemannschen Mannigfaltigkeit, Ann. Acad. Sci. Fenn. Ser A I no. 1 (1941).

M. OHTSUKA

1. Sur un théoréme etoilé de Gross, Nagoya Math. J. 9 (1955), 191-207.

BROWN UNIVERSTTY,

Providence, Rhode IsLAND 